\title{
MIR132 Pre-miRNA
}

National Cancer Institute

\section{Source}

National Cancer Institute. MIR132 Pre-miRNA. NCI Thesaurus. Code C82765.

MIR132 is an oligoribonucleotide that is encoded by the human MIR132 gene and has a role in the regulation of gene expression. 